\title{
ON THE EQUIVALENCE THEOREM IN THE $\chi P T$ DESCRIPTION OF THE SYMMETRY BREAKING SECTOR OF THE STANDARD MODEL
}

\author{
A. Dobadoq日 \\ Department of Physics, Stanford University \\ Stanford, CA 94305-4060, USA \\ and \\ J. R. Peláez 阿 \\ Departamento de Física Teórica II \\ Universidad Complutense de Madrid \\ 28040 Madrid, Spain \\ SU-ITP-93-33, hep-ph/9401202 \\ November 1993 \\ Extended version \\ April 1994
}

\begin{abstract}
We develop an alternative formulation of the symmetry breaking sector of the Standard Model as a gauged non-linear sigma model (NLSM) following the philosophy of the Chiral lagrangian approach, which is the only compatible with all the experimental and theoretical constraints. We derive the BRS symmetry of the model and the corresponding quantum lagrangian, which is a generalization of the standard Faddeev-Popov method, in a way which is covariant with respect to the reparametrizations of the coset space of the NLSM. Then we use the BRS invariance of the quantum lagrangian to state the Equivalence Theorem for the renormalized $S$-matrix elements calculated as a chiral expansion.
\end{abstract}

\footnotetext{
${ }^{1}$ On leave of absence from Departamento de Física Teórica, Universidad Complutense de Madrid, 28040 Madrid, Spain

${ }^{2}$ E-mail: dobado@cernvm.cern.ch

${ }^{3}$ E-mail: pelaez@fis.ucm.es
} 


\section{Introduction}

In the last years, a great deal of work has been devoted to the so called Chiral Perturbation Theory $(\chi P T)$ description of the symmetry breaking sector of the Standard Model (SM). Originally, $\chi P T$ [1] was developed for the description of hadronic physics at low energies where perturbative Quantum Cromodynamics (QCD) cannot be directly applied. $\chi P T$ is based in the approximate $S U(2)_{L} \times S U(2)_{R}$ symmetry of the hadronic interactions which is supposed to be spontaneously broken to the $S U(2)_{L+R}$ (isospin) subgroup. The corresponding Goldstone bosons (GB) are identified with the pions and their dynamics is obtained as a derivative expansion with couplings that have to be fitted from the experiments but that one could, at least in principle, compute from the underlying theory, i.e. QCD.

In the case of the symmetry breaking sector of the SM things are quite different but still we have some analogies with the low-energy hadron dynamics. Despite the very accurate precision measurements of the electroweak parameters recently performed at the Large Electron-Positron Collider (LEP), it is very few what is really known about the physical mechanism responsible for the breaking of the $S U(2)_{L} \times U(1)_{Y}$ gauge symmetry to the electromagnetic group $U(1)_{e m}$ (see [2] for pedagogical review). Basically, what we know is the following: There must be a system coupled to the SM with some global symmetry $G$ which is spontaneously broken through some undetermined mechanism to some subgroup $H$. This global symmetry breaking drives the spontaneous breaking of the gauge symmetry $S U(2)_{L} \times U(1)_{Y}$ to $U(1)_{e m}$ of the SM responsible for the $W^{ \pm}$and $Z$ masses. Therefore, the only established modes of that system are the three GB corresponding to the global symmetry breaking $G$ to $H$. Finally, these degrees of freedom will appear related through the Higgs mechanism to the longitudinal components of the massive vector bosons.

Apart from these very general considerations we also have some quantitative information about the symmetry breaking sector of the SM like the value of the dimensional constant $v \simeq 250 \mathrm{GeV}$ appearing in the low-energy matrix element between the GB and the gauge currents which can be obtained (using the gauge symmetry of the SM) from the muon lifetime. In addition we have the values of the gauge boson masses and the $\rho$ parameter which is known to be very close to one. It has been argued that the presence in the symmetry breaking sector of the SM of the so called custodial symmetry $S U(2)_{L+R}$ [3] as a subgroup of $H$ yields $\rho=1$ when considering only the contribution from this sector. The corrections coming from the coupling with the gauge bosons are in general small and compatible with the present experimental value of $\rho$.

Basically, this is all we really know about the symmetry breaking sector of the SM. Of course, in the literature one can find a huge number of proposals for the description of this sector, being the most well known the minimal SM (MSM) with a Higgs doublet and a potential defined ad hoc to produce the symmetry breaking, Technicolor (TC) [4] where one uses a similar mechanism to that producing the rupture of the chiral symmetry in QCD, and Supersymmetry (SUSY) (see [5] for a review) where, apart from the MSM fields, we have at least an extra doublet of Higgs fields and all their corresponding SUSY partners.

Having no idea of the method used by nature to give masses to the electroweak bosons, it seems to be quite reasonable to look for a model independent description of the GB low energy dynamics using nothing more than the well known facts discussed 
above. It is here then where the use of the $\chi P T$ methods become extremely useful. The first application of $\chi P T$ including the effect of GB loops to this context [6] dealt with the description of the elastic scattering of the gauge bosons longitudinal components, but more recently it has also been used to parametrize the precision tests of the SM coming from LEP [7].

Concerning the first kind of application, the so called Equivalence Theorem (ET) plays an essential role. This theorem states that, for renormalizable or $R_{\xi}$ gauges and at high energies compared with the gauge boson mass, the $S$-matrix elements for the GB are given by the corresponding $S$-matrix elements for the longitudinal components of the gauge boson (in the following we will call them generically $W_{L}$ ). The ET is usually assumed in many phenomenological descriptions of the elastic $W_{L} W_{L}$ scattering independently of the nature of the symmetry breaking sector of the SM. However, despite there are many heuristic arguments suggesting that some version of this theorem should apply in the general case, not only there is no proof of this fact but even there are some indications pointing that the concrete form of the theorem depends on the way the computations of the $S$-matrix are done. The first version of the theorem was given by Cornwall, Levin and Tiktopoulos and also by Vayonakis [8] at the tree level and it was extended at any order in the Feynman 't Hooft gauge by Lee, Quigg and Thacker for the case of one leg [9]. Later on, Chanowitz and Gaillard gave a complete and very systematic proof of the theorem valid at any order and for any number of legs [10] using the Ward-Slavnov-Taylor identities derived from the BecchiRouet-Stora (BRS) symmetry [11] of the SM. Further, this demonstration was slightly simplified by Gounaris, Kogerler and Neufeld [12]. Later, it was realized by Yao and Yuang [13] that renormalization affects differently the GB and the gauge bosons, and so produces corrections to the ET which in principle depend on the renormalization scheme and the gauge choice. Recently some articles have appeared trying to clarify this and other issues related with the ET [14].

In any case, it must be stressed that most of the work done on the ET refers exclusively to the MSM, and therefore, there are no solid reasons to apply this theorem when other symmetry breaking sectors or mechanisms are considered. In this paper we will try to fill this gap by obtaining the version of the ET that applies when the symmetry breaking sector of the SM is described by a general chiral lagrangian. The path we will pursuit to that end is roughly the following: First we will develop in detail the most general description of the GB dynamics compatible with the real information we have on the symmetry breaking sector of the SM. This will be done as in $\chi P T$ i.e. using a $S U(2)_{L} \times U(1)_{Y}$ gauged non-linear sigma model (NLSM) derivative expansion based on the coset $G / H$ so that all the information (or better the lack of information) about the GB dynamics will be parametrized by the couplings of the higher derivative terms. We will show how it is possible to do that independently of the coordinates used to parametrize the coset space $G / H$ so that the model only depends on $G$ and $H$. Then we will find the BRS transformations corresponding to the $S U(2)_{L} \times U(1)_{Y}$ symmetry of the gauged NLSM and we will obtain a BRS invariant quantized lagrangian using a generalization of the standard Faddeev-Popov method which gives rise to a well defined propagator for the gauge bosons, and therefore is appropriate for doing perturbative computations. The next step is the introduction of the renormalized lagrangian which is also BRS invariant due to the absence of anomalies in the SM. Then, as BRS invariance is a key ingredient of the formal Chanowitz-Gaillard proof of the ET we will be able 
to follow many of their steps also in our case. However, as we will see, some important differences arise due to the renormalization factors and the peculiarities of the $\chi P T$ description of the SM symmetry breaking sector.

Thus, the plan of the paper goes as follows. In sec.2 we define, according to the present experimental information, the NLSM based on the coset $G / H$ describing the dynamics of the GB that appear in the SM symmetry breaking sector, discussing its main properties both at the classical and at the quantum level. In sec.3 we introduce the $S U(2)_{L} \times U(1)_{Y}$ gauge degrees of freedom by gauging in the directions of the appropriate $G$ killing vectors to find the gauged NLSM which describes the interactions of the GB and the gauge bosons. Starting from the gauge transformations of the gauged NLSM we introduce in sec.4 the corresponding BRS and anti-BRS transformations building up the (anti-) BRS invariant quantum lagrangian appropriate for perturbative computations in $R_{\xi}$ gauges. In sec.5 we introduce the renormalized lagrangian and the renormalized BRS and anti-BRS transformations which leave it invariant when it is written in terms of renormalized fields. Then, in sec.6, and following the steps of the Chanowitz-Gaillard proof, we make use of the BRS invariance to obtain Ward-SlavnovTaylor Identities between renormalized Green functions containing gauge bosons and GB. In sec.7 we translate these relations to the $S$-matrix elements obtained through the chiral expansion. Section 8 is devoted to discuss the real meaning of our result and the existence of an energy region where $\chi P T$ and the ET can be safely used simultaneously. Finally, in sec.9 we briefly review the main results of this paper.

\section{The dynamics of the GB and its geometrical in- terpretation}

The most general description of the GB dynamics compatible with the information we have on this sector of the SM can be obtained as follows. First we turn off the gauge fields $\left(g=g^{\prime}=0\right)$. The rest of the system must have some global symmetry $G$ which is spontaneously broken to some subgroup $H$ through some unknown mechanism. We will represent by $T^{a}$ the generic hermitian $G$ generators with $a=1,2, \ldots, g=\operatorname{dim} G$. They can also be written as $T^{a}=H^{a}$ for $a=1,2 \ldots, h=\operatorname{dim} H$ and $T^{a}=X^{a}$ for $a=h+1, h+2, \ldots, g$ where the $H^{a}$ are the generators of $H$. We will assume $G$ to be compact and the coset space $K=G / H$ to be symmetric. This means that the commutator of two $X$-like generators will be a linear combination of $H$-like generators only.

It is possible then to define an involutive automorfism into $G$ as follows. Let $u \in G$ so that we can write $u=\exp i\left(\alpha_{a} H_{a}+\beta_{a} X_{a}\right)$, and the result of the automorfism acting on $u$, let us call it $u^{\prime}$, is obtained from $u$ by changing the sign of the parameters corresponding to the $X$-like generators, i e. $u^{\prime}=\exp i\left(\alpha_{a} H_{a}-\beta_{a} X_{a}\right)$. So defined, the automorfism is obviously involutive and it has $H$ as the maximal invariant subgroup. From the physical point of view, the meaning of this automorfism is the parity transformation which seems to play a central role in the electroweak interactions.

According to the Nambu-Goldstone theorem, the GB fields take values on the coset space $K=G / H$, so that there are just $k=\operatorname{dim} K=\operatorname{dim} G-\operatorname{dim} H=g-h$ of them. In order to parametrize the GB fields we need some coordinates $\omega^{\alpha}$ with $\alpha=1,2, \ldots, k$ on the coset $K$. Then the group $G$ can be understood as the isometry group of the 
coset $K$ when it is equipped with metrics built up as follows: Let $p$ be some point of $K$ with coordinates $\omega^{\alpha}$ and $u=1+i T^{a} \epsilon^{a}$ some small $G$ transformation. Then $u$ maps the point $p$ in another point $p^{\prime}$ of coordinates $\omega^{\prime \alpha}=\omega^{\alpha}+\delta \omega^{\alpha}$ so that we can write:

$$
\delta \omega^{\alpha}=\xi_{a}^{\alpha}(\omega) \epsilon^{a}
$$

This relation precisely defines how the $G$ symmetry is realized non-linearly by the GB fields $\omega^{\alpha}(x)$. From the geometrical point of view, $\xi^{a}=\xi_{a}^{\alpha} \partial / \partial \omega^{\alpha}$ can be interpreted as the killing vectors corresponding to the $G$ transformations acting on $K$. Now it is possible to define the $K$ metrics through the vielbein $e_{a}=e_{a}^{\alpha} \partial / \partial \omega^{\alpha}$ with $e_{a}^{\alpha}=\xi_{a+h}^{\alpha}$ for $a=1,2, \ldots, k$ i.e. the vielbein is just the set of killing vectors corresponding to the $k$ broken generators. The $K$ metrics $g_{\alpha \beta}$ is defined as the inverse of $g^{\alpha \beta}$ where:

$$
g^{\alpha \beta}=e_{a}^{\alpha} e^{\beta a}
$$

with $a=1,2, \ldots, k$. In the following, $\alpha, \beta, \ldots$ will refer to curved coordinates on $K$ and $a, b, \ldots$ to flat or tangent space indices. These indices are therefore raised and lowered with the tensors $g_{\alpha \beta}$ and $\delta_{a b}$ respectively. Now it is not difficult to show that the $G$ transformations are isometries of this metric, i.e.

$$
g_{\alpha \beta}^{\prime}(\omega)=g_{\alpha \beta}(\omega)
$$

so that, the metric functional dependence of the coordinates on $K$ does not change after the $G$ transformation.

With the above defined metric on $K$ it is possible to introduce the GB dynamics intrinsically, or in other words, independently of the choice of the coordinates on the coset space $K$, and therefore, only dependent on $G$ and $H$. The most general $G$ invariant lagrangian describing the low-energy dynamics of the GB can be organized by taking into account the number of derivatives of the different terms which must be covariant not only in the space-time sense but also in the $K$ sense. For example, the first term has two derivatives and can be written as:

$$
\mathcal{L}_{0}=\frac{1}{2} g_{\alpha \beta}(\omega) \partial_{\mu} \omega^{\alpha} \partial^{\mu} \omega^{\beta}
$$

The form of this lagrangian does not depend on the coordinates chosen on $K$ since it is $K$ covariant, and it is $G$ invariant too as can be easily checked using eq.3. Note also that the lagrangian not only contains the kinetic term but also all the infinite interaction terms that can be obtained by expanding the $K$ metrics $g_{\alpha \beta}$ in powers of $\omega^{\alpha}$ with an arbitrary (even) high number of GB fields .

Any other term that could be added to $\mathcal{L}_{0}$ to write the most general $K$ covariant and $G$ invariant lagrangian will have more derivatives so that we just write:

$$
\begin{aligned}
\mathcal{L} & =\mathcal{L}_{0}+c_{1}\left(g_{\alpha \beta}(\omega) \partial_{\mu} \omega^{\alpha} \partial^{\mu} \omega^{\beta}\right)^{2}+c_{2}\left(g_{\alpha \beta}(\omega) \partial_{\mu} \omega^{\alpha} \partial^{\nu} \omega^{\beta}\right)^{2} \\
& + \text { higher derivative terms }
\end{aligned}
$$

and so on. The constants appearing in these terms make it possible to parametrize systematically the GB dynamics of any theory with spontaneous symmetry breaking pattern from $G$ to $H$. 
Once the classical lagrangian has been defined we can go to the quantum theory by using standard path integral methods. The only subtlety that we have to take into account in this case is the following: Any quantum theory is not uniquely defined by the classical lagrangian but one also has to define a measure in the corresponding functional field space. Therefore, in order to have a $G$ invariant and $K$ covariant quantum theory not only do we need a lagrangian but also a measure with this properties. Then, as it has been discussed in the literature (see for instance [15]), the proper definition of the generating functional is:

$$
e^{i W[J]}=\int[d \omega \sqrt{g}] e^{i \int d^{n} x\left(\mathcal{L}\left(\omega, \partial^{2} \omega, \partial^{4} \omega, \ldots\right)+J_{a} \Gamma^{a}\right)}
$$

where $g$ is the determinant of the $K$ metrics, $n$ is the space-time dimension and $\Gamma^{a}$ is defined as follows: Given the point $p$ of $K$ with coordinates $\omega^{\alpha}$ and some other point 0 selected as the origin (the classical vacuum) with coordinates $\omega^{\alpha}=0$ we consider the geodesic curve joining 0 and $p$ (we assume both points are close enough for this geodesic to be unique). Now let $S$ be the distance between 0 and $p$ along the geodesic, with it we define $\Gamma_{\alpha}=\partial S / \partial \omega^{\alpha}$, which is then a vector, and $\Gamma^{a}=e^{\alpha a} \Gamma_{\alpha}$. With those definitions, the external source $J^{a}$ transforms like a vector and the whole eq. 6 becomes covariant.

When the $\sqrt{g}$ factor in the measure is re-exponenciated it gives rise to a new term in the lagrangian of the form:

$$
\Delta \mathcal{L}=-\frac{i}{2} \delta^{n}(0) \operatorname{tr} \log g
$$

The $\delta^{n}(0)$ can be written as $\int d^{n} k /(2 \pi)^{n}$, for this reason it is extremely convenient to use dimensional regularization when dealing with this kind of models, since it is well known that in this scheme one formally takes the space-time dimension to be $n=4-\epsilon$ and the integral vanishes. Then one can simply forget about the measure factor in eq. 6 . Of course, we could choose to work in other regularization schemes, in that case the contribution of the $\delta^{n}(0)$ term in the lagrangian will cancel other contributions which are also absent in the dimensional regularization scheme [16].

The main properties of the Green functions derived from the generating functional in eq. 6 are the following:

a) Although the amplitudes depend in general on the coordinates defined on $K$, the $S$-matrix elements do not, provided the new coordinates are related to the old ones as $\omega^{\alpha}=\omega^{\alpha}+f^{\alpha}(\omega)$ with $f$ being analytical and $f(0)=0$ i.e. the vacuum corresponds to $\omega^{\alpha}=0$ in any coordinate system. Then the predictions that can be made on physical process with the model are independent of the coordinates chosen on $K$.

b) The $G$ invariance of the (regularized) $W[J]$ functional gives rise to the corresponding Ward-Slavnov-Taylor identities which in this case are called the low-energy theorems since they can predict the very low-energy dynamics of the GB.

c) The counterterms needed to absorb the divergences are also $G$ invariant. As far as all these terms are included in $\mathcal{L}$ with their corresponding couplings $c_{i}$, the theory is renormalizable in this generalized sense. The only difference with other much more conventional theories such as $Q C D$ is that here we have an infinite number of coupling constants. However, following the original philosophy of the chiral lagrangians, since one is interested in the low-energy dynamics it is enough to compute the Green 
functions only to some given power of the external momenta. In this case, only a finite number of terms and couplings contribute, so that, at this level, the theory is completely predictive.

Now the question arises on which is the proper choice of the groups $G$ and $H$ defining the quotient space $K=G / H$ for the description of the GB modes of the symmetry breaking sector of the SM. The conditions that these groups should satisfy are the following:

a) As we need three GB to give mass to the three observed gauge bosons $W^{ \pm}$and $Z$ we have $k=\operatorname{dim} K=g-h=3$.

b) $G$ must contain the group $S U(2)_{L} \times U(1)_{Y}$ in order to be able to couple the symmetry breaking sector to the electroweak gauge boson.

c) In order to ensure the experimental relation $\rho \simeq 1$ we require the custodial symmetry to be present i.e. the subgroup $H$ must contain the custodial group $S U(2)_{L+R}$. This automatically yields $\rho=1$ when the gauge fields are switched off and also implies that the photon will remain massless since $U(1)_{e m}$ is contained in $S U(2)_{L+R}$ and therefore in $H$.

In principle one could think that the above conditions on the $G$ and $H$ groups are not very restrictive but we will show that there is only one possible choice of these two groups compatible with them. This can be seen as follows: The maximum number of isometries of some given $k$-dimensional space is $k(k+1) / 2$ (see for instance [17]). In our case this number must be larger or equal than the dimension of the isometry group $G$ i.e.:

$$
\frac{k(k+1)}{2} \geq g=k+h
$$

But condition a) requires $k=3$ and condition c) means $h \geq 3$ so that $6 \geq 3+h \geq 6$ which implies $h=3$ and then $H=S U(2)_{L+R}$. In addition $g=6$ and taking into account condition b) we arrive to the conclusion that $G=S U(2)_{L} \times S U(2)_{R}$ is the only possibility. Thus $K=G / H$ is just $S^{3}$ i.e. the three dimensional sphere. In the following we will concentrate in these choices for $G$ and $H$ which is the only consistent with conditions a),b) and c) and therefore the only relevant for the description of the symmetry breaking sector of the SM as a NLSM.

Once we know which is the quotient space we have to work with, namely $S^{3}$, one can ask which are the more suitable coordinates to parametrize it. In principle, the formalism that we are going to use is completely covariant and can be used independently of the precise coordinate choice. However, the most commonly used are the following:

1) Standard coordinates, where:

$$
g_{\alpha \beta}=\delta_{\alpha \beta}+\frac{\omega^{\alpha} \omega^{\beta}}{v^{2}-\omega^{2}}
$$

being $v$ the radius of the sphere (this magnitude corresponds to the vacuum expectation value of the Higgs fields in the MSM so we have $v \simeq 250 \mathrm{GeV}$ ). These coordinates are obtained just imposing on the standard flat four dimensional space of Cartesian coordinates $\left(\omega^{1}, \omega^{2}, \omega^{3}, \sigma\right)$ the spherical constraint $\omega^{2}+\sigma^{2}=v^{2}$.

2) Chiral coordinates where the fact that $S U(2)=S^{3}$ is used to ensemble the GB fields in a single $S U(2)$ matrix $U(x)=\exp \left(i \omega^{\alpha} \sigma^{\alpha} / 2 v\right)$. In this case the metrics is given by:

$$
g_{\alpha \beta}=\frac{-v^{2}}{4} \operatorname{tr} \frac{\partial U}{\omega^{\alpha}} \frac{\partial U^{\dagger}}{\omega^{\beta}}
$$


3) Finally, sometimes it is useful to use geodesic coordinates where $\omega^{\alpha}=\Gamma^{\alpha}$. In these coordinates the coupling of the external source to the GB fields in eq. 6 becomes especially simple and they are the most natural from the geometrical point of view. In any case, it is important to remark that even if one does not use geodesic coordinates we always have $\omega^{\alpha}=\Gamma^{\alpha}+O\left(\omega^{2}\right)$

The use of one or another of the above coordinate systems can make some of the computations much easier in different situations. Nevertheless, as stressed before, in further developments we will use a covariant formalism which will be valid for any coordinate choice provided all them are analytical and keep the classical vacuum at $\omega^{\alpha}=0$.

\section{Gauging the NLSM}

Once the NLSM describing the GB dynamics has been defined, the next step is to switch on the gauge fields. This can be done as usual by turning the derivatives of the NLSM into covariant derivatives and adding the pure Yang-Mills terms for the gauge fields. We define the covariant derivative through those $G$ Killing vectors which correspond to the gauge group $S U(2)_{L} \times U(1)_{Y}$. As discussed in the previous section, these Killing vectors are obtained from the transformation equations for the GB fields. For the $S U(2)_{L} \times U(1)_{Y}$ transformations we have:

$$
\begin{array}{r}
\delta_{L} \omega^{\alpha}(x)=l_{a}^{\alpha} \epsilon_{L}^{a}(x) \\
\delta_{Y} \omega^{\alpha}(x)=y^{\alpha} \epsilon_{Y}(x)
\end{array}
$$

where $l_{a}^{\alpha}$ with $a=1,2,3$ are the Killing vectors corresponding to the $S U(2)_{L}$ group and $y^{\alpha}$ corresponds to $U(1)_{Y}$. In fact, $y^{\alpha}$ is just the third killing vector of the group $S U(2)_{R}$. Now, the covariant derivative for the GB fields is defined as;

$$
D_{\mu} \omega^{\alpha}=\partial_{\mu} \omega^{\alpha}-g l_{a}^{\alpha} W_{\mu}^{a}-g^{\prime} y^{\alpha} B_{\mu}
$$

where $W_{\mu}^{a}$ and $B_{\mu}$ are the $S U(2)_{L}$ and $U(1)_{Y}$ gauge fields and $g$ and $g^{\prime}$ are the corresponding gauge couplings. Note that the gauge fields are vectors both in the Minkowsky space and in the $S^{3}$ sense. Thus the lagrangian of the gauged NLSM can be written as;

$$
\begin{aligned}
\mathcal{L}_{g} & =\mathcal{L}_{Y M}^{L}+\mathcal{L}_{Y M}^{Y} \\
& +\frac{1}{2} g_{\alpha \beta}(\omega) D_{\mu} \omega^{\alpha} D^{\mu} \omega^{\beta} \\
& + \text { higher covariant derivative terms }
\end{aligned}
$$

where $\mathcal{L}_{Y M}^{L}$ and $\mathcal{L}_{Y M}^{Y}$ are the Yang-Mills lagrangians for the $S U(2)_{L}$ and $U(1)_{Y}$ gauge fields.

The above lagrangian is invariant under the $S U(2)_{L}$ and $U(1)_{Y}$ gauge transformations:

$$
\begin{aligned}
\delta \omega^{\alpha} & =l_{a}^{\alpha} \epsilon_{L}^{a}(x)+y^{\alpha} \epsilon_{Y}(x) \\
\delta W_{\mu}^{a} & =\frac{1}{g} \partial_{\mu} \epsilon_{L}^{a}(x)+\epsilon_{a b c} W_{\mu}^{b} \epsilon_{L c}(x) \\
\delta B_{\mu} & =\frac{1}{g^{\prime}} \partial_{\mu} \epsilon_{Y}(x)
\end{aligned}
$$


provided the killing vectors $l_{a}=l_{a}^{\alpha} \partial / \partial \omega^{\alpha}$ and $y=y^{\alpha} \partial / \partial \omega^{\alpha}$ satisfy:

$$
\begin{aligned}
\left\{l_{a}, l_{b}\right\} & =\epsilon_{a b c} l_{c} \\
\left\{l_{a}, y\right\} & =0 \\
\{y, y\} & =0
\end{aligned}
$$

which are the so called closure relations. Here $\{x, y\}$ should be understood as the Lie brackets of the Killing vectors $x$ and $y$ i.e.:

$$
\{x, y\}=\frac{\partial y^{\alpha}}{\partial \omega^{\gamma}} x^{\gamma}-\frac{\partial x^{\alpha}}{\partial \omega^{\gamma}} y^{\gamma}
$$

The closure relations are required to ensure that the $l_{a}$ and $y$ Killing vectors are a realization of the $S U(2)_{L}$ and $U(1)_{Y}$ symmetries on the coset manifold $S^{3}$. Since we are using a covariant formalism all the time, we only need to check these relations for some particular coordinate choice on $S^{3}$. For example, for standard coordinates it is not difficult to show:

$$
\begin{aligned}
l_{a}^{\alpha} & =-\frac{1}{2}\left(\epsilon_{\alpha a \gamma} \omega^{\gamma}-\delta_{\alpha a} \sqrt{v^{2}-\omega^{2}}\right) \\
y^{\alpha} & =-\frac{1}{2}\left(\epsilon_{\alpha 3 \gamma} \omega^{\gamma}-\delta_{\alpha 3} \sqrt{v^{2}-\omega^{2}}\right)
\end{aligned}
$$

With this expression for the $l_{a}$ and $y$ Killing vectors it is straightforward to check the closure relations in eq.15 directly and this is all we need to show the $S U(2)_{L} \times U(1)_{Y}$ gauge invariance of the lagrangian in eq.13 for any coordinate choice on $S^{3}$. Similarly, it is possible to state the Jacobi identity as follows: Let $\delta_{1}, \delta_{2}$ and $\delta_{3}$ be three arbitrary gauge transformations like that in eq.15 with different parameters. Then we have:

$$
\left[\delta_{\epsilon_{1}},\left[\delta_{\epsilon_{2}}, \delta_{\epsilon_{3}}\right]\right]+\left[\delta_{\epsilon_{3}},\left[\delta_{\epsilon_{1}}, \delta_{\epsilon_{2}}\right]\right]+\left[\delta_{\epsilon_{2}},\left[\delta_{\epsilon_{3}}, \delta_{\epsilon_{1}}\right]\right]=0
$$

where the gauge transformation applies both to the GB and the gauge fields.

Thus, starting from the most general lagrangian for the NLSM (the one in eq.5) it is possible to build an $S U(2)_{L} \times U(1)_{Y}$ gauged NLSM lagrangian just replacing, as usual, the derivatives by covariant derivatives and including the Yang-Mills terms for the gauge bosons. In addition, we could consider other terms like:

$$
F_{\mu \nu}^{a} F_{a}^{\mu \nu} g_{\alpha \beta}(\omega) D_{\rho} \omega^{\alpha} D^{\rho} \omega^{\beta}
$$

where $F_{\mu \nu}$ represents the standard stress tensor for the $S U(2)_{L}$ or the $U(1)_{Y}$ gauge fields. In fact, some of these terms, which are gauge invariant but not just obtained by the replacement of the derivatives by covariant derivatives in the NLSM lagrangian, are needed in the quantized theory to cancel some of the divergences coming from the original (gauged) NLSM. In any case, it must be stressed that, even when one only considers the terms with two covariant derivatives in eq.5, the $S U(2)_{L} \times U(1)_{Y}$ gauged NLSM is no longer globally $S U(2)_{L} \times S U(2)_{R}$ nor $S U(2)_{L+R}$ invariant. In particular, there are terms due to the $U(1)_{Y}$ gauge field which break these symmetries and that is why the $\rho$ parameter gets electroweak corrections that drive its value out of one when $g^{\prime}$ is taken to be different from zero, as it happens in the MSM. 
It is worth mentioning that in the NLSM formulation of the standard model considered here it is not necessary to go to unitary gauge to obtain the gauge bosons mass matrix since for any coordinate choice on $S^{3}$ we have:

$$
\begin{aligned}
l_{a}^{\alpha} & =\frac{v}{2} \delta_{a}^{\alpha}+O(\omega) \\
y^{\alpha} & =-\frac{v}{2} \delta_{3}^{\alpha}+O(\omega) \\
g_{\alpha \beta} & =\delta_{\alpha \beta+} O\left(\omega^{2}\right)
\end{aligned}
$$

and therefore, the $g^{\prime 2} g_{\alpha \beta} y^{\alpha} y^{\beta} B_{\mu} B^{\mu} / 2$ term appearing in eq.13 (inside the term with two covariant derivatives ) when expanded in powers of the GB fields yields a $g^{\prime 2} v^{2} B_{\mu} B^{\mu} / 4$ piece contributing to the mass matrix for any gauge and for any coordinate choice. However, we still have terms like $g g_{\alpha \beta} \partial^{\mu} \omega^{\alpha} W_{\mu}^{a} l_{\beta a} / 2$ which contains in its expansion the unwanted contribution $g^{2} v \partial^{\mu} \omega^{\alpha} W_{\mu \alpha} / 4$. Nevertheless, these mixing terms can be cancelled in the quantized theory using the t' Hooft gauge condition ( $R_{\xi}$-gauges).

To end this section, we will introduce some notations in order to save a lot of space in next sections by considering the $S U(2)_{L}$ and $U(1)_{Y}$ gauge groups at the same time. To do that we introduce the Killing vector $L_{a}^{\alpha}$ with $a=1,2,3,4$ as $L_{a}^{\alpha}=g l_{a}^{\alpha}$ for $a=1,2,3$ and $L_{4}^{\alpha}=g^{\prime} y^{\alpha}$. We also introduce the completely antisymmetric symbols $f_{a b c}$ with $a=1,2,3,4$ as $f_{a b c}=g \epsilon_{a b c}$ for $a=1,2,3$ and $f_{a b 4}=0$, and finally the gauge field $W_{\mu}^{a}$ with $a=1,2,3,4$ will be defined as $W_{\mu}^{a}=W_{\mu}^{a}$ for $a=1,2,3$ and $W_{\mu}^{4}=B_{\mu}$. With this notation the two covariant derivative term in eq.13 can be written as:

$$
\begin{aligned}
& \frac{1}{2} g_{\alpha \beta}(\omega) D_{\mu} \omega^{\alpha} D^{\mu} \omega^{\beta}= \\
& \quad \frac{1}{2} g_{\alpha \beta}(\omega) \partial_{\mu} \omega^{\alpha} \partial^{\mu} \omega^{\beta}-g_{\alpha \beta} \partial_{\mu} \omega^{\alpha} L_{\alpha a} W^{\mu a}+\frac{1}{2} g_{\alpha \beta} L_{a}^{\alpha} L_{b}^{\beta} W^{\mu a} W^{\mu b}
\end{aligned}
$$

therefore, the mass matrix is just the zeroth order term of $g_{\alpha \beta} L_{a}^{\alpha} L_{b}^{\beta}$ when expanded in powers of the GB fields. The closure relations in eq.15 can now be gathered in:

$$
\left\{L_{a}, L_{b}\right\}=f_{a b c} L^{c}
$$

\section{The BRS symmetry and the quantized lagrangian}

The following step of our program is to build a quantized version of our classical gauged NLSM appropriate for doing perturbative computations. When a renormalizable gauge theory is described with the fields belonging to linear representations of the gauge group and linear gauge fixing conditions, the Faddeev-Popov method using a standard 't Hooft gauge is very appropriate for this task providing a BRS invariant lagrangian from which we could derive the Ward-Slavnov-Taylor identities that will lead us to the ET.

However, in order to make the proof as general as possible, we are interested in building a Lagrangian covariant under changes of coordinates in the GB coset. As we will see, a crucial point in the proof of the ET is the usage of a 't Hooft gauge fixing function. Usually, this function depends linearly on the GB fields which are coordinates in the manifold that, in general, do not transform like vectors in the coset index, and therefore will not render a gauge fixing term covariant under reparametrizations. Thus, 
if we insist in demanding the lagrangian to be covariant under coordinate changes in the coset, the 't Hooft gauge fixing function should depend nonlinearly in the GB fields. It is known that Yang-Mills theories quantized with non-linear gauge fixing conditions generate radiatively quartic ghost interactions. That is why we will use the technique described in [18] that generalizes the Fadeev-Popov method and introduces naturally this quartic ghost terms through the BRS and anti-BRS invariance of the quantized lagrangian. It is important to remark that if we had chosen to work with a particular set of coordinates, the GB fields could have appeared linearly in the 't Hooft gauge fixing function, and then we could have used the standard Fadeev-Popov procedure, but that would not imply that the results could be translated directly to other coset coordinates.

The quantization method goes as follows: We start from a gauge invariant lagrangian like that considered in the previous section where the fields do not necessarily transform linearly. Then, by introducing anticommuting ghost fields one obtains the corresponding BRS and anti-BRS transformation (at this point the closure and Jacobi identities in eq.15, eq.18 and eq.22 play an decisive role). Next we build the so called quantum lagrangian, BRS and anti-BRS invariant, which is a generalization of the standard classical lagrangian plus the usual gauge fixing and Faddeev-Popov terms. Therefore, the Feynman rules and a well defined perturbative expansion for the gauged NLSM can be derived as it is usually done.

Thus we start introducing the ghost fields $c^{a}$ and $\bar{c}^{a}$ where the flat index $a=1,2,3$ refers to the $S U(2)_{L}$ ghost fields and $a=4$ refers to that from $U(1)_{Y}$. They are scalar fields and anticommuting Grassmann variables. Now, the BRS transformations for the $\mathrm{GB}$, gauge bosons and the ghost fields can be written as:

$$
\begin{aligned}
s\left[\omega^{\alpha}\right] & =L_{a}^{\alpha} c^{a} \\
s\left[W^{\mu a}\right] & =\partial^{\mu} c^{a}+f_{b c}^{a} W^{\mu b} c^{c} \equiv D_{a c}^{\mu} c^{c} \\
s\left[c^{a}\right] & =-\frac{1}{2} f_{b c}^{a} c^{b} c^{c}
\end{aligned}
$$

and the anti-BRS ones as:

$$
\begin{aligned}
\bar{s}\left[\omega^{\alpha}\right] & =L_{a}^{\alpha} \bar{c}^{a} \\
\bar{s}\left[W^{\mu a}\right] & =D_{a c}^{\mu} \bar{c}^{c} \\
\bar{s}\left[\bar{c}^{a}\right] & =-\frac{1}{2} f_{b c}^{a} \bar{c}^{b} \bar{c}^{c}
\end{aligned}
$$

and in addition, we define the combined relation;

$$
s\left[\bar{c}^{a}\right]+\bar{s}\left[c^{a}\right]=-f_{b c}^{a} \bar{c}^{b} \bar{c}^{c}
$$

Still we have to define the BRS transformation for the antighost field and the anti-BRS for the ghost field. To do that we introduce the auxiliary commuting scalar field $b^{a}$. Then we write:

$$
\begin{aligned}
& s\left[\bar{c}^{a}\right]=b^{a} \\
& s\left[b^{a}\right]=0
\end{aligned}
$$


and

$$
\begin{aligned}
& \bar{s}\left[\bar{c}^{a}\right]=-b^{a}-f_{b c}^{a} \bar{c}^{b} c^{c} \\
& \bar{s}\left[b^{a}\right]=-f_{b c}^{a} \bar{c}^{b} c^{c}
\end{aligned}
$$

This completes the set of BRS and anti-BRS transformations. The action of the BRS and the anti-BRS operators $s$ and $\bar{s}$ on more complex combinations of fields can be defined assuming that these are linear differential operators graded by the ghost number so that we have $s[X Y]=s[X] Y \pm X s[Y]$ where the minus sign applies if there is an odd number of ghost or anti ghost in the product of fields $X$ (note that the auxiliary field $b^{a}$ has zero ghost number).

With the above definition for the BRS and the anti-BRS operators it is possible to show that they are nilpotent in the following sense

$$
s^{2}=s \bar{s}+\bar{s} s=\bar{s}^{2}=0
$$

In order to show these nilpotency relations we have to verify that they hold for all the fields. The computations are tedious but straightforward provided one uses eq.23 eq. 24 , eq. 25 , eq. 26 and eq. 27 , the other properties of the $s$ and the $\bar{s}$ operators and the closure and Jacobi identities in eq. 15 or eq.22 but the details will not be given here. As the (anti-) BRS transformation properties for the physical fields (the GB and the gauge bosons) can be understood as gauge transformations where the gauge parameter is just the (anti-) ghost field, one could say that the original gauge transformations plus the closure and the Jacobi identities are equivalent to the (anti-) BRS transformation plus the nilpotency relations realized in the enlarged system formed by the physical fields, the (anti-) ghosts and the auxiliary field. However, as it is well known, the (anti-) BRS version of the gauge transformations is by far much more appropriate for the quantum theory, and in particular for perturbation theory. As a matter of fact, the physical Hilbert space can be defined as the cohomology of the BRS operator, i.e. as the set of states which are annihilated by the BRS operator not being the result of the action of the BRS operator on any other state. In addition, the complete antisymmetry of the $f_{b c}^{a}$ symbols ensures the existence of an invariant functional integral measure for the fields of the enlarged system.

Now we will define the quantum lagrangian as the most general one being covariant (in the space time and the $S^{3}$ sense), $s$ and $\bar{s}$ invariant and with ghost number zero . It can be written as:

$$
\mathcal{L}_{Q}=\mathcal{L}_{g}+\frac{1}{2} s \bar{s}\left[G\left(\omega^{\alpha}, W_{\mu}^{a}, c^{a}, \bar{c}^{a}, b^{a}\right)\right]
$$

where $G$ is some scalar function of all the fields with zero ghost number and $\mathcal{L}_{g}$ is the classical gauge invariant lagrangian (the factor $1 / 2$ is included for further convenience). The (anti-) BRS invariance of the above quantum lagrangian follows from the gauge form of these transformations for the physical fields and the nilpotency relations of the (anti-) BRS operator. Different choices of $G$ correspond to different gauge fixing functions for the quantized theory. In our case, we are dealing with a gauge theory which is spontaneously broken and for this reason the t' Hooft or renormalizable gauge fixing conditions ( $R_{\xi}$-gauges) are very appropriate. These kind of gauges have the two following essential properties; first they give rise in the quantum lagrangian to a bilinear 
term for the gauge fields which is invertible so that we have a well defined gauge field propagator that can be used in perturbation theory. Second, a piece appearing in the quantum lagrangian cancels the unwanted mixing term which appears in the classical lagrangian (inside the second term in the RHS of eq.21). In the framework of the BRS formalism considered here, the $R_{\xi}$-gauges can be obtained using the function:

$$
G=A W_{\mu}^{a} W^{\mu a}+B f(\omega)+C c^{a} \bar{c}_{a}
$$

where $f$ is some arbitrary scalar function on $S^{3}$ i.e. on the GB fields with;

$$
\frac{\partial f}{\partial \omega^{\alpha}}=\Gamma_{\alpha}+O\left(\omega^{2}\right)
$$

The constants $A, B$ and $C$ will be adjusted to cancel the unwanted mixing term coming from the classical lagrangian. Giving the appropriate values to these constants we obtain:

$$
\mathcal{L}_{Q}=\mathcal{L}_{g}+\frac{1}{2} s \bar{s}\left[W_{\mu}^{a} W^{\mu a}-2 \xi f(\omega)+\xi c^{a} \bar{c}_{a}\right]
$$

Now, working out the (anti-) BRS transformation we finally obtain the following quantum lagrangian;

$$
\begin{aligned}
\mathcal{L}_{Q} & =\mathcal{L}_{g}-\frac{1}{2 \xi}\left[\partial_{\mu} W^{\mu a}+\xi \frac{\partial f}{\partial \omega^{\alpha}} L^{\alpha a}\right]^{2} \\
& +D_{\mu b}^{a} c^{b} \partial^{\mu} \bar{c}_{a}-\xi\left\{\frac{\partial^{2} f}{\partial \omega^{\alpha} \partial \omega^{\beta}} L_{a}^{\alpha} L_{b}^{\beta}+\frac{\partial f}{\partial \omega^{\alpha}} \frac{\partial L_{a}^{\alpha}}{\partial \omega^{\beta}} L_{b}^{\beta}\right\} c^{b} \bar{c}^{a} \\
& +\frac{1}{2}[\bar{c}, c]_{a}\left(\partial_{\mu} W^{\mu a}+\xi \frac{\partial f}{\partial \omega^{\alpha}} L^{\alpha a}\right)+\frac{\xi}{4}[\bar{c}, c]^{2}
\end{aligned}
$$

where $c=T^{a} c^{a}$ and $\bar{c}=T^{a} \bar{c}^{a}$ being $T^{a}=\sigma^{a} / 2$ for $a=1,2,3$ and $T^{4}=1 / 2$ and $[c, \bar{c}]=[c, \bar{c}]^{a} T^{a}$. The gauge fixing function has appeared in the second term of the RHS of the above lagrangian since the $b$ field equation of motion is given by:

$$
b^{a}=\partial_{\mu} W^{\mu a}+\xi \frac{\partial f}{\partial \omega^{\alpha}} L^{\alpha a}
$$

so that the auxiliary $b^{a}$ plays the role of a Lagrange multiplier needed to implement the gauge fixing condition. The gauge fixing terms give rise to the standard $R_{\xi}$ propagator for the gauge fields and it is not difficult to check that it cancels the unwanted terms in the classical lagrangian using eq.31 and remembering that $\omega^{\alpha}=\Gamma^{\alpha}+O\left(\omega^{2}\right)$ for any coordinate system. The following four terms are the generalization of the standard Faddeev-Popov method including the kinetic part for the (anti-) ghost fields and their interactions with the gauge and the GB. Finally, we have the last contribution which produces quartic ghost interactions. This term cannot be obtained by the standard Faddeev-Popov procedure. However, it is radiatively generated in Yang-Mills theories quantized with non-linear gauge fixing functions. In our case, it comes from the $c \bar{c}$ piece in our $G$ function through a $b^{2}$ term (the gauge fixing condition), being a genuine element in the formalism used here.

In order to be sure of our results after applying the $s$ and the $\bar{s}$ operator to our $G$ function we have also checked directly that the whole quantum lagrangian in eq.33 is (anti-) BRS invariant as it obviously should be. 
Thus we have succeeded in the construction of a quantum lagrangian corresponding to the the most general gauged NLSM describing the dynamics of the GB and the gauge fields. The Feynman rules to be used in perturbation theory can be derived as usual from this quantum lagrangian. Of course, the precise form of these rules depends on the coordinates one chooses in the coset $S^{3}$. However, our quantum lagrangian is completely covariant and (anti-) BRS invariant and it is independent of the coordinates and the gauge provided it is renormalizable. At this point it is worth noting that for the particular choice of the Landau gauge, which corresponds to take $\xi=0$, the quantum lagrangian in eq.33 becomes greatly simplified and, in particular, there are no interactions between the (anti-) ghost and the GB fields.

\section{The renormalized lagrangian}

Now we can derive the Ward-Slavnov-Taylor identities for the dimensionally regularized Green functions that are needed in the proof of the ET, making use of the BRS invariance of the lagrangian in eq.33. It is important to remark that dimensional regularization is used in order to avoid the $-\frac{i}{2} \delta^{n}(0) t r \log g$ contribution from the path integral measure of the GB fields, as well as to preserve the (anti)-BRS invariance in the regularized lagrangian.

However, for practical purposes we are interested in the renormalized Green functions in order to make predictions for the different physical processes. The Green functions obtained from the lagrangian in eq.33 present divergencies that have to be cancelled by considering a renormalized lagrangian made of the original "bare" lagrangian of eq.33 plus the counterterms needed to reproduce the whole set of divergent structures. Although these counterterms have been given only up to those with four derivatives [19], all them should also be (anti)-BRS invariant, since if this was not the case the model would be anomalous, i.e., the gauge invariance of the model would be broken by quantum effects. However, the standard hypercharge assignments in the SM, and the fact that the number of colors is $N_{c}=3$, ensures the cancellation, generation by generation, of all the possible gauge and mixed gauge-gravitational anomalies, including the non-perturbative $S U(2)$ discovered by Witten [20]. Therefore, even though we coupled chiral fermions to the NLSM the resulting theory would be free of these anomalies. Apart from the (anti)-BRS invariance, the model we have built is also invariant under reparametrizations of the GB, since it has been shown in [21] that the potential anomalies that could break the invariance under changes of coordinates on the coset, are absent from the NLSM when it is defined in spaces with a dimension smaller than that of the space-time.

Therefore, although the (anti)-BRS invariant lagrangian obtained when taking into account all the needed counterterms is made of an infinite number of terms, it can be understood as the renormalized lagrangian of a renormalizable theory with an infinite number of couplings written in terms of bare quantities. As it happens in any renormalizable theory, we can also write this renormalized lagrangian by means of renormalized fields and couplings, so that the terms which appear now will keep the same form (due to the fact that the theory is renormalizable in the sense described above) although they present some $Z$ factors. The relation between bare and renormalized fields and gauge couplings is given by: 


$$
\begin{gathered}
W_{0 \mu}^{a}(x)=Z_{3}^{(a) 1 / 2} W_{\mu}^{a}(x) ; \omega_{o}^{\alpha}(x)=Z_{\omega}^{(\alpha) 1 / 2} \omega^{\alpha}(x) ; g_{0}^{(a)}=Z_{g}^{(a)} g^{(a)} ; \xi_{0}^{(a)}=Z_{3}^{(a)} \xi^{(a)} \\
c_{0}^{a}(x)=\widetilde{Z}_{2}^{(a) 1 / 2} c^{a}(x) ; \bar{c}_{0}^{a}(x)=\widetilde{Z}_{2}^{(a) 1 / 2} \bar{c}^{a}(x) ; B_{0}^{a}(x)=\widetilde{Z}_{2}^{(a)} B^{a}(x) ; v_{0}=Z_{v}^{1 / 2} v
\end{gathered}
$$

where $g^{(a)}=g$ for $a=1,2,3$ and $g^{(4)}=g^{\prime}$. The first three $Z_{3}$ are the same thanks to the gauge structure of the model. As a notation, in the following we will use that the indices between parenthesis are not summed and that those fields and constants without 0 subscripts are renormalized. We have also introduced for further convenience the $B$ field which is the $b$ auxiliary field with a different normalization: $B_{0}^{a}=\sqrt{\xi_{0}} b_{0}^{a}$. In addition, the symmetries of the chiral lagrangian provide infinite relations between the bare and the renormalized couplings. Indeed, thanks to the (anti)-BRS invariance of the renormalized lagrangian given in terms of the bare quantities, it is possible to find the following "renormalized" (anti)-BRS transformations which will leave invariant the renormalized lagrangian once it is written in terms of the renormalized fields and couplings:

$$
\begin{array}{ll}
s_{R}\left[\omega^{\alpha}\right]=X^{(a)} L_{R a}^{\alpha} c^{a} & \bar{s}_{R}\left[\omega^{\alpha}\right]=X^{(a)} L_{R a}^{\alpha} \bar{c}^{a} \\
s_{R}\left[W^{\mu a}\right]=X^{(a)} D_{R c}^{\mu a} c^{c} & \bar{s}_{R}\left[W^{\mu a}\right]=X^{(a)} D_{R c}^{\mu a} \bar{c}^{c} \\
s_{R}\left[c^{a}\right]=-\frac{X^{(a)}}{2} f_{R b c}^{a} c^{b} c^{c} & \bar{s}_{R}\left[c^{a}\right]=-\frac{X^{(a)}}{2} f_{R b c}^{a} \bar{c}^{b} c^{c} \\
s_{R}\left[\bar{c}^{a}\right]=X^{(a)} \frac{B^{a}}{\sqrt{\xi^{(a)}}} & \bar{s}_{R}\left[\bar{c}^{a}\right]=-X^{(a)}\left(\frac{B^{a}}{\sqrt{\xi^{(a)}}}+f_{R b c}^{a} \bar{c}^{b} c^{c}\right) \\
s_{R}\left[B^{a}\right]=0 & \bar{s}_{R}\left[B^{a}\right]=-X^{(a)} f_{R b c}^{a} \bar{c}^{b} B^{c}
\end{array}
$$

where $L_{R a}^{\alpha}=Z_{\omega}^{(\alpha)-1 / 2} Z_{3}^{(a) 1 / 2} L_{a}^{\alpha}, f_{R b c}^{a}=Z_{g}^{(a)} Z_{3}^{(a) 1 / 2} g f_{b c}^{a}$, and $X^{(a)}=\widetilde{Z}_{2}^{(a) 1 / 2} / Z_{3}^{(a)}$.

Once we have a set of (anti)-BRS symmetry transformations for the renormalized fields, and a renormalized lagrangian which is invariant under such transformations, we can obtain Ward-Slavnov-Taylor identities for the renormalized Green functions by means of the standard functional methods. In particular, we are going to use some of these relations to find the actual formulation of the ET that holds when the symmetry breaking sector of the SM is described through the chiral lagrangian formalism.

\section{Ward-Slavnov-Taylor Identities}

As discussed in the introduction, our aim in this section is to use the general functional procedures to obtain Ward-Slavnov-Taylor identities showing the relationship between renormalized Green functions involving an arbitrary number of longitudinal gauge bosons $W_{L}$, with those Green functions where we have replaced all the external $W_{L}$ by their corresponding GB. In order to do that we will basically follow the same steps of the Chanowitz-Gaillard proof of the ET.

In the preceding section, we have explicitly built an (anti-) BRS invariant renormalized quantum lagrangian for the $S U(2)_{L} \times U(1)_{Y}$ gauged NLSM. Now we can use it to obtain Ward-Slavnov-Taylor identities involving Green functions which will lead 
us to the desired relations for $S$ matrix elements. To do so, we start from the standard identity derived from the lagrangian BRS invariance (here $A$ stands for any of the renormalized fields appearing in the quantum lagrangian i.e. $\left.A_{i}=\omega^{\alpha}, W_{\mu}^{a}, c^{a}, \bar{c}^{a}, B^{a}\right)$ :

$$
\sum_{i} \int d^{4} x<s_{R}\left[A_{i}\right]>_{J} J_{i}(x)=0
$$

Where the BRS transformations are made of linear and nonlinear products of fields, so that, in general, we can write :

$$
<s_{R}\left[A_{i}\right]>_{J}=\sum_{n} s_{A_{i}}^{i_{1} \ldots i_{n}}<A_{i_{1}} \ldots A_{i_{n}}>_{J}
$$

In most of the cases we only have to take into account the linear terms, but when $A_{i}=W^{\mu}, c^{a}$ we have to consider products of two fields, and when $A_{i}=\omega^{\alpha}$ all the infinite terms that appear due to the nonlinear realization of the symmetry. Now we can relate these expectation values with the generating functional for connected Green functions $W_{R}\left(x_{1}, \ldots, x_{n}\right)$, using the standard functional formalism:

$$
<A_{i_{1}} \ldots A_{i_{n}}>_{J}=\frac{\delta^{(n)} W_{R}[J]}{\delta J_{i_{1}} \ldots \delta J_{i_{n}}}
$$

where, as usual, $J_{i}$ is the external current associated with the $A_{i}$ field, and the generating functional is given by:

$$
\begin{aligned}
W_{R}[J] & =\sum_{n=1} \int d^{4} x_{1} d^{4} x_{2} \ldots d^{4} x_{n} W_{R i_{1}, \ldots, i_{n}}\left(x_{1}, x_{2}, \ldots, x_{n}\right) J_{i_{1}}\left(x_{1}\right) \ldots J_{i_{n}}\left(x_{n}\right) \\
& =(2 \pi)^{4} \sum_{n=1} \int \prod_{i=1}^{n} \frac{d^{4} p_{i}}{(2 \pi)^{4}} \delta^{4}\left(\sum_{i} p_{i}\right) J_{i_{1}}\left(-p_{1}\right) \ldots J_{i_{n}}\left(-p_{n}\right) W_{R i_{1}, \ldots, i_{n}}\left(p_{1}, \ldots p_{n}\right)
\end{aligned}
$$

(from now on we will be working in momentum space). Using the preceding relations, the condition of BRS invariance in eq.37 becomes:

$$
I[J]=\sum_{i} \sum_{n} s_{A_{i}}^{i_{1} \ldots i_{n}} \int \frac{d^{4} q d^{4} k_{1} \ldots d^{4} k_{n-1}}{(2 \pi)^{4 n}} \frac{\delta^{(n)} W_{R}[J]}{\delta J_{i_{1}}\left(q-k_{1}\right) \ldots \delta J_{i_{n}}\left(k_{n-1}\right)} J_{i}(-q)=0
$$

A similar equation can be written for the renormalized anti-BRS transformations too. It is straightforward then to obtain Ward-Slavnov-Taylor identities from the last formula just by taking functional derivatives with respect to $J_{i}(p)$ and setting then $J=0$. In particular, since we want to relate external $W_{L}$ with GB, we are interested in identities involving the auxiliary $B$ field because, as we have seen yet, when using its equation of motion it gives the gauge fixing condition which is hinting us the possible relation between longitudinal vector bosons and GB. If we look again to the BRS transformations, we find that the $B$ field only appears in $s_{R}[\bar{c}]$, and as we are looking for a relation where all the $W_{L}$ are replaced by $\omega$, we need to derive functionally once by $\bar{c}$.

As an example, and since we will need the result for the general proof, we are going to obtain a relation with just one $B$ and another arbitrary field. Besides, it illustrates some differences with the proof given by Chanowitz-Gaillard. We start from:

$$
\left.\frac{\delta}{\delta J_{\bar{c}_{b}}(-k)} \frac{\delta}{\delta J_{j}(p)} I[J]\right|_{J=0}=0
$$


Noticing that the only possible contributions come from $A_{j}=W^{\mu}$ or $A_{j}=\omega^{\alpha}$, we obtain the following relations:

$$
\begin{aligned}
& X^{(a)}\left(L_{R a}^{\alpha(0)}+\Delta_{2 a}^{\alpha}\left(p^{2}\right)\right) W_{c^{a} \bar{c}^{b}}(p)+\frac{X^{(b)}}{\sqrt{\xi^{(b)}}} W_{B^{b} \omega^{\alpha}}(p)=0 \\
& X^{(a)} i p_{\mu}\left(1+\Delta_{3}\left(p^{2}\right)\right) W_{c^{a} \bar{c}^{b}}(p)+\frac{X^{(b)}}{\sqrt{\xi^{(b)}}} W_{B^{b} W_{\mu}^{a}}(p)=0
\end{aligned}
$$

Where we have expanded $L_{R a}^{\alpha}$ as:

$$
L_{R a}^{\alpha}=L_{R a}^{\alpha(0)}+L_{R a}^{\alpha \beta(1)} \omega^{\beta}+L_{R a}^{\alpha \beta \gamma(2)} \omega^{\beta} \omega^{\gamma}+\ldots
$$

and we have used the following definitions:

$$
\begin{aligned}
i p_{\mu} \Delta_{3}\left(p^{2}\right) & =f_{R d c}^{a} W_{c^{a} \bar{c}^{b}}^{-1}(p) \int \frac{d^{4}}{(2 \pi)^{4}} W_{W^{\mu d} c^{c} \bar{c}^{b}}(p-q, q, p) \\
\Delta_{2 a}^{\alpha}\left(p^{2}\right) & =L_{R a}^{(1) \alpha \beta} W_{c^{c} \bar{c}^{b}}^{-1}(p) \int \frac{d^{4}}{(2 \pi)^{4}} W_{\omega^{\beta} c^{c} \bar{c}^{b}}(p-q, q, p)+\ldots
\end{aligned}
$$

Therefore we can gather these results in:

$$
\frac{X^{(b)}}{\sqrt{\xi^{(b)}}} W_{B^{b} l}(p)=-X^{(a)} D_{R l}^{a}(p) W_{c^{a} \bar{c}^{b}}(p)
$$

where:

$$
D_{R l}^{a}(p)=i p_{\mu}\left(1+\Delta_{3}\left(p^{2}\right)\right) \delta_{l}^{W_{\mu a}}+\left(L_{R a}^{(0) \alpha}+\Delta_{2 a}^{\alpha}\left(p^{2}\right)\right) \delta_{l}^{\omega^{\alpha}}
$$

and in the last step we have introduced the $D_{R l}^{a}(p)$ operator which will play a similar role to the $D_{l}^{a}(p)$ operator defined by Chanowitz and Gaillard, although we can see yet some important differences with their formal proof: First, the $L_{R}^{(0)}$ factor coming from the nonlinear realization of the symmetry, which appears only in its zeroth order and in the end will be the only remainder of the complicated relation between gauge and Goldstone bosons that one naively expects from the nonlinear gauge fixing condition. Second, the $X$ factors due to renormalization. And third, the $\Delta$ terms that were correctly introduced by Bagger and Schmidt [14] in the context of the MSM.'.

Now, in order to derive the general expression we start from:

$$
\left.\frac{\delta}{\delta J_{\bar{c}_{a_{1}}}(-k)} \prod_{j=2}^{s} \frac{\delta}{\delta J_{B_{a_{j}}}\left(-k_{j}\right)} \prod_{k=1}^{m} \frac{\delta}{\delta J_{A_{k}}\left(-p_{k}\right)} I[J]\right|_{J=0}=0
$$

Where we will impose to the $J_{A_{k}}$ currents to be associated to physical $A_{k}$ fields only. Therefore, as we are not taking functional derivatives with respect to $J_{\omega}$ nor $J_{c}$, we can easily see from the BRS transformations that we do not get any contribution when $A_{i}=B$, neither when $A_{i}=\omega, c$. Since the $A_{k}$ are physical, their polarization vectors satisfy $\epsilon \cdot k_{\mu}=0$ and thus they will cancel the first term coming from $s_{R}\left[W_{\mu}^{a}\right]=$

\footnotetext{
${ }^{4}$ Recently we have noticed [22] a paper where these $\Delta$ terms were correctly introduced in the chiral lagrangian formalism
} 
$i k_{\mu} c^{a}+\epsilon_{R b c}^{a} W_{\mu b} c_{c}$. Therefore we only have to take into account those contributions coming from $s_{R}[\bar{c}]$ and the part which is left from $s_{R}\left[W_{\mu}^{a}\right]$ that will be called generically, "bilinear terms". Finally we can write:

$$
\frac{X^{\left(a_{1}\right)}}{\sqrt{\xi^{\left(a_{1}\right)}}} W_{B_{a_{1}} B_{a_{2}} \ldots B_{a_{s}} A_{1} \ldots A_{m}}\left(k_{1}, \ldots, k_{s}, p_{1}, \ldots p_{m}\right)+\text { bilinear terms }=0
$$

where $\sum_{i} k_{i}=-\sum_{i} p_{i}$. In order to translate this result to off-shell $S$-matrix elements, we apply the Lehmann-Symanzik-Zimmermann (LSZ) reduction formula which yields:

$$
\begin{aligned}
& \frac{X^{\left(a_{1}\right)}}{\sqrt{\xi^{\left(a_{1}\right)}}}\left(\prod_{i=1}^{m} W_{A_{i} A_{i}}\left(p_{i}\right)\right) \sum_{l_{j}}\left(\prod_{j=1}^{s} W_{B_{a_{j} l_{j}}}\left(k_{j}\right)\right) S_{l_{1} . . l_{s} A_{1} \ldots A_{m}}^{o f f-s h e l l}\left(k_{1} \ldots k_{s}, p_{1} \ldots p_{m}\right) \\
& + \text { bilinear terms }=0
\end{aligned}
$$

Note that the $a_{1}$ index is not contracted so that the factor $X / \sqrt{\xi}$ is not relevant and we can drop it. The next step to obtain $S$-matrix elements is to multiply by the inverse renormalized propagators of the $A_{i}$ fields the whole eq.50. When we set their momenta on shell, that is $p_{i}^{2}=m_{A_{i}}^{2}$, we can cancel the "bilinear terms" since they contain a Green function made of more than $s+m$ fields, so that one of the momenta is off-shell, and therefore the pole needed to compensate for $W_{A_{i} A_{i}}^{-1}\left(p_{1}\right) \rightarrow 0$ is absent and the term vanishes as $p_{1}^{2} \rightarrow m_{A_{1}}^{2}$. Finally, we can use eq.46 to substitute the $B$ field two point functions, and then write:

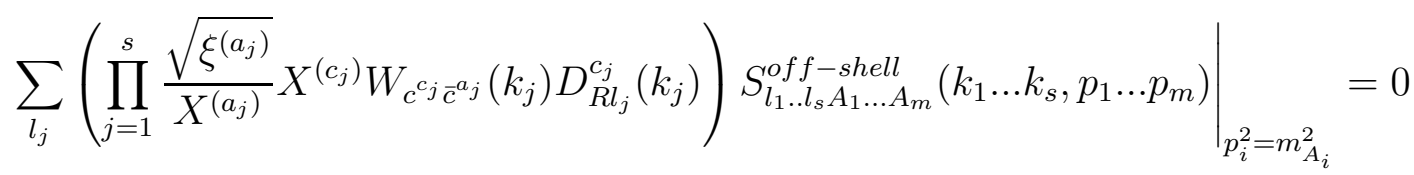

Again, the $a_{j}$ are not contracted, and therefore, we can take away the $\sqrt{\xi^{\left(a_{j}\right)}} / X^{a_{j}}$ factors. Now, although the renormalized ghost two point function may be in principle non-diagonal, we can multiply by its inverse $W_{c^{d_{j} \bar{c}^{a_{j}}}}^{-1}\left(k_{j}\right)$ so that the $d_{j}$ index is set free thus allowing us to drop the last $X$ factor. Therefore, we arrive to the following expression:

$$
\left.\sum_{l_{1} \ldots l_{r}} \prod_{i=1}^{s} D_{R l_{i}}^{a_{i}}\left(p_{i}\right) S_{l_{1} . . l_{s}, A_{1} . . A_{m}}^{\text {off-shell }}\left(p_{1} . . p_{s}, k_{1} . . k_{m}\right)\right|_{k_{i}^{2}=m_{A_{i}}^{2}}=0
$$

\section{The Equivalence Theorem}

Our aim is now to complete the LSZ procedure to obtain from eq.52 relations between $S$-matrix elements by setting all the momenta on-shell. However, before doing so, we have to obtain the physical field combinations since they are not the $W_{\mu}$ fields in the $D_{R}$ operator. This step is achieved by means of a transformation $\widetilde{W}_{\mu}^{a}=R^{a b} W_{\mu}^{b}$, which can be given in the most general form by:

$$
\left(\begin{array}{c}
\widetilde{W}_{\mu}^{1} \\
\widetilde{W}_{\mu}^{2} \\
\widetilde{W}_{\mu}^{3} \\
\widetilde{W}_{\mu}^{4}
\end{array}\right)=\left(\begin{array}{c}
W_{\mu}^{-} \\
W_{\mu}^{+} \\
Z_{\mu}^{\text {phys }} \\
A_{\mu}^{\text {phys }}
\end{array}\right)=\left(\begin{array}{cccc}
1 / \sqrt{2} & i / \sqrt{2} & 0 & 0 \\
1 / \sqrt{2} & -i / \sqrt{2} & 0 & 0 \\
0 & 0 & \cos \theta & -\sin \theta \\
0 & 0 & \sin \theta^{\prime} & \cos \theta^{\prime}
\end{array}\right)\left(\begin{array}{c}
W_{\mu}^{1} \\
W_{\mu}^{2} \\
W_{\mu}^{3} \\
W_{\mu}^{4}
\end{array}\right)
$$


The renormalized fields thus obtained are precisely those which ensure that their exact propagators have poles located at the right values of their corresponding physical masses. Similarly we also define: $\widetilde{L}_{R \alpha}^{(0) b}=L_{R \alpha}^{(0) a}\left(R^{-1}\right)^{b a}$, as well as $\widetilde{\Delta}_{2 a}^{\alpha}$, and so we write:

$$
\sum_{l_{1} \ldots l_{r}} \prod_{i=1}^{s} \widetilde{D}_{R l_{i}}^{a_{i}}\left(p_{i}\right) S_{l_{1} . . l_{s}, A_{1} . . A_{m}}\left(p_{1} . . p_{s}, k_{1} . . k_{m}\right)=0
$$

where

$$
\widetilde{D}_{R l}^{a}(p)=i p_{\mu}\left(1+\widetilde{\Delta}_{3}\left(M_{p h y s}^{2}\right)\right) \delta_{l}^{\widetilde{W}_{R \mu a}}+\left(\widetilde{L}_{R a}^{(0) \alpha}+\widetilde{\Delta}_{2 a}^{\alpha}\left(M_{p h y s}^{2}\right)\right) \delta_{l}^{\omega^{\alpha}}
$$

Note that we have already set the $p_{i}$ momenta on-shell for the massive physical vector bosons i.e. $p_{i}^{2}=M_{i}^{2}$ phys. Thus this will also be the on-shell conditions for the GB in the final version of the ET.

The next step is now to convert the momentum factors of $\widetilde{D}_{R l}^{a}(p)$ in longitudinal polarization vectors $\epsilon_{(L)}=p_{\mu} / M_{\text {phys }}+v_{\mu}$ since we expect to neglect the $v_{\mu}$ four-vector at sufficiently high energies because $v_{\mu} \simeq O\left(M_{\text {phys }} / E\right)$. Unfortunately, this step is not straightforward. The gauge structure of the theory will produce cancellations in the amplitudes involving longitudinally polarized gauge bosons, and we are not allowed to simply drop out the terms containing $v_{\mu}$ vectors.

The way around this problem is to perform a power counting analysis to extract the relevant orders for the ET statement. It will be very convenient to derive from eq.54 an expression involving only longitudinal polarization vectors and $v_{\mu}$ factors, but without any momentum multiplying the amplitudes. Such an expression was first obtained in [10] and, although we have introduced the $K_{R}$, its derivation is completely analogous. For the sake of completeness, we will sketch it here. We start defining:

$$
V(l, n, m)=\left(\prod_{i=1}^{n} v_{\mu_{i}}\right)\left(\prod_{j=1}^{l} K_{\alpha_{j}}^{a_{j}}\right) S_{\omega^{\alpha_{1}} \ldots \omega^{\alpha} ; ; \widetilde{W}_{R \mu_{1}}^{b_{1}} \ldots \widetilde{W}_{R \mu_{n}}^{b_{n}} ; A_{1} \ldots A_{m}}(p ; q ; k)
$$

where we have introduced $K_{R a}^{\alpha}=\left(\widetilde{L}_{R a}^{(0) \alpha}+\widetilde{\Delta}_{2 a}^{\alpha}\left(M_{\text {phys }}^{(a) 2}\right)\right) /\left(M_{\text {phys }}^{(a)}\left(1+\widetilde{\Delta}_{3}\left(M_{\text {phys }}^{(a) 2}\right)\right)\right)$, which reflects the effects of the renormalization procedure and of the non-linear realization of the theory, and thus, up to here, are the main difference with the ET proof given in [10]. It will be important to remark that these factors do not depend on the energy. f

If we write $v_{\mu}=\epsilon_{(L)}-p_{\mu} / M_{p h y s}$ we will obtain a sum whose generic term with $s$ longitudinal polarization vectors and $n-s$ momentum factors will be of the general form:

$$
\begin{aligned}
& X(l, n-s, s, m)=\left(\prod_{i=1}^{n-s} \frac{q_{\mu_{i}}}{M_{b_{i}}}\right)\left(\prod_{k=1}^{s} \epsilon_{(L)}^{\nu_{k}}\left(r_{k}\right)\right) \times \\
& \left(\prod_{j=1}^{l} K_{\alpha_{j}}^{a_{j}}\right) S_{\omega^{\alpha_{1} \ldots \omega^{\alpha} l} ; \widetilde{W}_{R \mu_{1}}^{b_{1}} \ldots \widetilde{W}_{R \mu_{n-s}}^{b_{n-s}} ; \widetilde{W}_{R \nu_{1}}^{c_{1}} \ldots \widetilde{W}_{R \nu_{s}}^{c_{s}} A_{1} \ldots A_{m}}(p ; q ; r ; k)
\end{aligned}
$$

Indeed, when writing explicitly $V(l, n, m)$, these $X(l, n-s, s, m)$ terms will appear with different contractions of indices, so that we can write:

$$
\bar{V}(l, n, m)=\sum_{s=0}^{n}(-1)^{n-s} \bar{X}(l, n-s, s, m)
$$

\footnotetext{
${ }^{5}$ When we were completing this work we noticed [22] a paper where the renormalization factors have been considered in the ET for the chiral lagrangian formalism
} 
where the bar means a sum over all independent permutations of the indices $\left(a_{i}, p_{i}\right)$, $\left(b_{i}, q_{i}\right)$, and $\left(c_{k}, r_{k}\right)$.

With these notations it is easy to see that eq.54 can be written as:

$$
\sum_{l=0}^{n-s}(-i)^{n-s-l} \bar{X}(l, n-s-l, s, m)=0
$$

We can multiply this last equation by $i^{s}$, adding the result from $s=0$ to $s=n-1$. Thus we obtain:

$$
\begin{aligned}
0 & =\sum_{s=0}^{n-1} i^{s} \sum_{l=0}^{n-s}(-i)^{n-s-l} \bar{X}(l, n-s-l, s, m) \\
& =\sum_{l=0}^{n} i^{n-l} \sum_{s=0}^{n-l}(-1)^{n-s-l} \bar{X}(l, n-s-l, s, m)-i^{n} \bar{X}(0,0, n, m)
\end{aligned}
$$

There is only one permutation in $\bar{X}(0,0, n, m)$ so that we can drop the bar, and using eq.58 we finally arrive to:

$$
X(0,0, n, m)=\sum_{l=0}^{n}(-i)^{l} \bar{V}(l, n-l, m)
$$

which is the desired result. When dealing with the formalism of chiral lagrangians it is more frequent to use amplitudes than $S$ matrix elements, and so we will do from now on. It is straightforward then to rewrite our last result as follows:

$$
\begin{aligned}
\left(\prod_{i=1}^{n} \epsilon_{(L) \mu_{i}}\right) & T\left(\widetilde{W}_{a_{1}}^{\mu_{1}}, \ldots, \widetilde{W}_{a_{n}}^{\mu_{n}} ; A\right)= \\
= & \sum_{l=0}^{n}(-i)^{n-l}\left(\prod_{i=1}^{l} v_{\mu_{i}}\right)\left(\prod_{j=l+1}^{n} K_{\alpha_{j}}^{a_{j}}\right) \bar{T}\left(\widetilde{W}_{a_{1}}^{\mu_{1}} \ldots \widetilde{W}_{a_{l}}^{\mu_{l}}, \omega_{\alpha_{l+1}} \ldots \omega_{\alpha_{n}} ; A\right)
\end{aligned}
$$

(notice that $l$ stands now for the number of gauge bosons, instead of the number of GB).

When one is dealing with amplitudes which satisfy the unitarity bounds, one is sure that they will never grow with the energy and, due to the fact that $v_{\mu} \simeq O\left(M_{\text {phys }} / E\right)$, then it is possible to neglect at high energies all terms in the RHS of eq.62 but that with $l=0$, which is precisely the one where all the external $\widetilde{W}_{L}$ have been substituted by GB. Although these considerations are general, in practice, the amplitudes are obtained through $\chi \mathrm{PT}$ as a truncated series in powers of the energy. They satisfy unitarity just in the perturbative sense, and thus the same reasoning is not valid. We have to use power counting methods to extract the leading contributions.

In our case, the power counting analysis goes as follows: We can, in principle, make a formal Laurent expansion in $E / 4 \pi v$ of the amplitudes, but as they should satisfy the Low Energy Theorems (second reference in [3]) in the $M^{2} \ll E^{2}$ regime, we can always write the negative energy powers as $(M / E)^{k}$. However, in the most interesting applications there will only be even negative powers since they come from an even number of polarization vectors multiplied by the energy expansion of propagators. In practice, one typically chooses the maximum positive power $N$ of the energy appearing 
in the computations when fixing the maximum number of derivatives in the lagrangian terms, so that we can write:

$$
\begin{aligned}
\bar{T}\left(\widetilde{W}_{a_{1}}^{\mu_{1}} \ldots \widetilde{W}_{a_{l}}^{\mu_{l}}, \omega_{\alpha_{l+1}} \ldots \omega_{\alpha_{n}} ; A\right) & \simeq \sum_{k=0}^{N} a_{l}^{k}\left(\frac{E}{4 \pi v}\right)^{k}+\sum_{k=1}^{\infty} a_{l}^{-k}\left(\frac{M}{E}\right)^{k} \\
\left(\prod_{i=1}^{n} \epsilon_{(L) \mu_{i}}\right) T\left(\widetilde{W}_{a_{1}}^{\mu_{1}}, \ldots, \widetilde{W}_{a_{n}}^{\mu_{n}} ; A\right) & \simeq \sum_{k=0}^{N} b^{k}\left(\frac{E}{4 \pi v}\right)^{k}+\sum_{k=1}^{\infty} b^{-k}\left(\frac{M}{E}\right)^{k}
\end{aligned}
$$

where we have set $g^{\prime}=0$ momentarily. This series are formal and the coefficients $a_{l}^{k}, b^{k}$ (for the sake of brevity we have omitted their field indices) could contain logarithms or further dependence in $g$. Therefore, in addition to the derivative expansion we can now make perturbative calculations on $g$ so that we can write $a_{l}^{h}=a_{l L}^{h}(1+O(g / 4 \pi))$ where $a_{l L}^{h}$ is the lowest order term in the expansion of $a_{l}^{h}$ in powers of $g$. Similarly, and due to the fact that in most renormalization schemes we have $M \simeq M_{\text {phys }}(1+O(g / 4 \pi))$, we can expand $K$ too. That is: $K_{\alpha}^{a} \simeq K_{\alpha}^{a(0)}+K_{\alpha}^{a(1)}(g / 4 \pi)+\ldots$. When these series are introduced in eq.62 we arrive to the following expression:

$$
\begin{aligned}
& \left(\prod_{i=1}^{n} \epsilon_{(L) \mu_{i}}\right) T\left(\widetilde{W}_{a_{1}}^{\mu_{1}}, \ldots, \widetilde{W}_{a_{n}}^{\mu_{n}} ; A\right) \simeq \\
& \simeq\left(\prod_{j=1}^{n} K_{\alpha_{j}}^{a_{j}(0)}\right) \sum_{k=0}^{N-n}\left(a_{0 L}^{k}(1+O(g / 4 \pi))\right)\left(\frac{E}{4 \pi v}\right)^{k}+O\left(\frac{M}{E}\right)+O\left(\frac{E}{4 \pi v}\right)^{N-n+1}
\end{aligned}
$$

Where we have neglected terms of order $O(M / E)$ and $O(E / 4 \pi v)^{N-n+1}$, and we have kept only the lowest order in the $g$ expansion of the energy coefficients. This is the statement of the ET valid for the chiral lagrangian description of the SM. Note that we are approximating the chiral expansion coefficients of the amplitude with all the longitudinal gauge bosons, not by the corresponding coefficients of the amplitude with all the $W_{L}$ substituted by GB, but by their lowest order in the $g$ or $g^{\prime}$ expansion. The validity of the ET only to the lowest order in $g$ has been already suggested in the literature (see the first cite in [14]), although in reference to the complete amplitudes, not for each coefficient in the truncated chiral expansions.

In order to check the last expression, we have explicitly computed the tree level amplitudes (so that all $Z$ factors are equal to one) up to four derivatives obtained from the lagrangian of the second reference in [19] for two processes, $Z_{L}^{0} Z_{L}^{0} \rightarrow Z_{L}^{0} Z_{L}^{0}$ and $W_{L}^{+} W_{L}^{-} \rightarrow Z_{L}^{0} Z_{L}^{0}$ using chiral coordinates for the coset $S^{3}$. The computation was done in two ways; first we have calculated the amplitude for the corresponding gauge bosons and we have proyected them into their longitudinal components. Then those $S$ matrix were compared with the GB $S$ matrix elements to zeroth order in $g$ and $g^{\prime}$ (see later the discussion about $g^{\prime}$ different from zero) and we found a perfect agreement at high energies (above about $1 \mathrm{TeV}$ ) both analytical and numerically, for different values of the chiral coupling constants (although of course, in the large energy regime, the fourth derivative approximation is not expected to be appropriate due to the bad energy behavior and the lack of unitarity of the amplitudes).

Turning to the general case, and for practical purposes, if we want all the approximations considered above to be reasonable, the allowed values of the energy should fall into the following applicability window:

$$
M \ll E \ll 4 \pi v=4 \pi M / g
$$




$$
g / 4 \pi \ll(E / 4 \pi v)^{N-n+1}
$$

The first two inequalities come from neglecting the $O(M / E)$ and $O(E / 4 \pi v)^{N-n+1}$ terms respectively. The last constraint is needed to reconcile both high and low energy requirements, since we are taking into account the $O(E / 4 \pi v)^{N-n}$ contribution while neglecting that of $O(M / E)$.

Now, the generalization to the case when $g^{\prime} \neq 0$ is simple since $g^{\prime} \ll g$ as well as $M_{Z}^{\text {phys }} \simeq M_{W}^{\text {phys }} \simeq M^{(a)}$ for any $a$ ( all the different masses are of the same order when counting energy powers). Thus if we keep the lowest order in the $g$ or $g^{\prime}$ expansion of the $a$ coefficients the approximation is still valid. Therefore, we can use eq.64 as the precise statement of the ET for the $\chi \mathrm{PT}$ description of the SM.

It is important to remark that this discussion in terms of energy expansions, is due to the fact that at high energies the effective lagrangian does not yield a good unitary behavior for the truncated amplitudes (as it happens in standard $\chi P T$ ), that could grow indefinitely [23]. That possibility does not allow us to consider only the $v_{\mu}=O(M / E)$ factors when extracting the leading energy term in eq.62, since the amplitudes can contain positive powers of $E$. However, if we could implement an unitary approximation, then the unitarized amplitudes at high energies will never grow with a power of $E$, and then we will be allowed to simply drop the terms with $v_{\mu}$ factors, thus obtaining:

$$
\left(\prod_{i=1}^{n} \epsilon_{(L) \mu_{i}}\right) T\left(\widetilde{W}_{a_{1}}^{\mu_{1}}, \ldots, \widetilde{W}_{a_{n}}^{\mu_{n}} ; A\right) \simeq\left(\prod_{j=1}^{n} K_{\alpha_{j}}^{a_{j}}\right) T\left(\omega_{\alpha_{1}} \ldots \omega_{\alpha_{n}} ; A\right)+O(M / E)
$$

which is the usual formal statement of the ET. This unitarization procedure can be achieved, for instance, by means of the Padé approximants and dispersion relations [23], large N-limit [24], etc..., and they could enlarge considerably the ET applicability range.

Finally we would like to mention that another trivial example in which the version of the ET in eq.66 can be applied is in the MSM considered as a particular case of the general models analysed in this work. This is so since the effective action obtained once the Higgs field is formally integrated out, is a gauged NLSM of the kind here considered, although all the couplings are now functions of the Higgs mass (see for instance [25] for a computation of the coefficients corresponding to the four derivatives terms). In addition, if one does not integrate the Higgs field, the theory is renormalizable in the standard sense, thus yielding a good unitary and high energy behavior and therefore eq.66 can be safely applied.

\section{Discussion}

In the previous section we finally arrived to the version of the ET valid for the gauged NLSM describing the GB and the gauge boson interactions in the symmetry breaking sector of the SM. This theorem relates the $S$-matrix elements for the GB with those of the longitudinal components of the GB $W_{L}$ at high energies. The demonstration is based in the Ward-Slavnov-Taylor identities coming from the BRS symmetry of the quantized lagrangian and applies for any $R_{\xi}$-gauge and for any choice of the coset coordinates or GB fields. The different renormalization of the GB and the gauge fields 
appearing in the gauge fixing condition produces some new factors that were absent in the original versions of the ET.

Still one could ask about the real utility of the ET in the gauged NLSM or $\chi P T$ description of the symmetry breaking sector of the SM. In principle there is an apparent contradiction in the terms of the title of this work, since $\chi P T$ provides a low-energy description of the GB dynamics as an expansion on the momenta over $4 \pi v$ and the ET refers to the large energy relation between the GB and the $W_{L}$ 's $S$-matrix elements. Of course, the relevant point is whether there exists an applicability window for the theorem in the intermediate energy region of eq.65 where $\chi P T$ and the ET could be safely applied simultaneously. For example, for the important case of the elastic scattering of longitudinal gauge bosons the lowest bound obtained from the second relation in eq.65 is equal to $1.7 \mathrm{TeV}$ when the chiral expansion is made considering terms with four derivatives. This suggests that for the standard one-loop computation the ET applicability range would be $1.7 \mathrm{TeV}<<E<<4 \pi v \simeq 3.1 \mathrm{TeV}$. This window is in principle narrow but note that it is located in a energy region where one-loop $\chi P T$ is not a very good approximation (for the case of the elastic pion scattering it corresponds roughly to $0.68 \mathrm{GeV}<<E<<1 \mathrm{GeV}$ ). In any case, only detailed computations of concrete scattering processes can give us positive information about the existence, the position and the width of the window for the simultaneous application of standard $\chi P T$ and the ET.

However, even when the applicability range of, let say, one-loop $\chi P T$ and the ET were too small to be useful, the description given here of the symmetry breaking sector of the SM as a gauged NLSM and the corresponding ET could be quite useful. This is because, apart from the perturbative one-loop (or more) computations, there are non-perturbative procedures to extend the $\chi P T$ to higher energies. These techniques include the use of the dispersion relations [23] and the large $N$ limit [24], $N$ being the number of GB. These two methods work very well in the case of hadron physics (specially the first one) and are also expected to do so in the case of the symmetry breaking sector of the SM, where they could be combined safely with the ET.

Therefore we have in principle three different ways to apply the ET depending on the kind of theory and the precise method used for making computations: First we can consider the case of a renormalizable theory in the standard sense with a finite number of parameters as, for example, the MSM. In this case the good high energy behavior of the $S$ matrix elements is granted and we arrive to the ET as stated in eq.66 without an upper energy bound. For the practical purposes the only problem is the computation the the GB $S$ matrix elements and the $K$ factors which depend on the the gauge and the renormalization prescriptions. The second scenario to be considered is when we use standard $\chi P T$ to describe the SBS of the standard model and we truncate, as usual, the chiral series at some order in the number of derivatives. In this case the precise statement of the theorem is that of eq.64 although only in the applicability window of eq.65. This version of the theorem is weaker, but in this case the computation of the $K$ factors is not so hard as in the previous one since we only need to know the lowest order in their $g$ and $g^{\prime}$ perturbative expansion. Finally the SBS of the SM could also be described by means of $\chi P T$ but making the computations in a non-standard way using some kind of non-perturbative approximation like those discussed above so that we have a good high energy behavior of the $S$ matrix elements. In this case, the version of the ET is that of eq.66 without an upper energy bound or applicability window but 
including the $K$ factors.

\section{Conclusion}

We will now briefly resume the main results of the present work: First, following the philosophy of $\chi P T$, we have built the gauged NLSM based on the coset $S^{3}=$ $S U(2)_{L} \times S U(2)_{R} / S U(2)_{L+R}$ describing the GB and the gauge boson dynamics of the symmetry breaking sector of the SM using all the experimental information we have about this sector without further assumptions. This description is done covariantly, i.e., it is independent of the coordinates selected to parametrize the coset manifold and for the above reasons it can be considered as the most general model independent description of the SBS of the SM.

Then we have built the renormalized quantum lagrangian in the $R_{\xi}$-gauges which

are appropriate for doing perturbative computations. This renormalized lagrangian is invariant under a set of renormalized (anti-) BRS transformations and this fact can be used to find the corresponding Ward-Slavnov-Taylor identities for the renormalized Green functions, which previously have been regularized dimensionally.

These Ward-Slavnov-Taylor identities were used to find a version of the ET holding for the renormalized $S$-matrix elements. The final version of this theorem has two important differences with the original one for the MSM: First, it includes renormalization corrections which in general will depend on the renormalization conditions and the gauge choice (the $K$ factors). Second, when one works with the standard $\chi P T$ truncated energy expansions, it does not relate directly the $S$-matrix elements with longitudinal gauge bosons to those with GB but it relates the respective coefficients in both expansions to the lowest order in $g$ or $g^{\prime}$.

In principle, the energy window for the simultaneous application of $\chi P T$ at the one-loop level and the ET can be narrow but non-perturbative methods could be used to extend it to higher energy regions thus providing a model independent description of the symmetry breaking sector dynamics. We sincerely believe that this kind of approach is the most sensible and realistic, until no further information about this sector is available, and in particular, it can provide a very useful phenomenological tool to manage the precision test of the SM provided by LEP and the future Large Hadron Collider (LHC) physics in an unified model independent scheme.

\section{Acknowledgements}

This work has been supported in part by the Ministerio de Educación y Ciencia (Spain)(CICYT AEN90-0034). We are very grateful to M. Urdiales for helping us checking explicitly the ET, and C.P.Martin for helpful suggestions. A.D. also thanks the Gregorio del Amo Foundation (Universidad Complutense) for support and S. Dimopoulos and the Department of Physics of the Stanford University for their kind hospitality. 


\section{Note Added}

When this work was being completed we noticed the appearance of two related preprints on the subject. In [22] the authors arrive, using a different quantization procedure, to similar results to us for $g^{\prime}=0$ and the GB parametrization $U=\exp \left(i \sigma^{a} \pi^{a} / v\right)$. They concentrate in the renormalization factors which correct the ET version without the power counting analysis. See also [26] for a discussion on this last issue.

\section{References}

[1] S. Weinberg, Physica 96A (1979) 327

J. Gasser and H. Leutwyler, Ann. of Phys. 158 (1984) 142, Nucl. Phys. B250 (1985) 465 and 517

[2] M.S. Chanowitz, Ann. Rev. Nucl. Part. Sci. 38 (1988) 323

[3] P. Sikivie et al., Nucl. Phys. B173 (1980) 189

M. S. Chanowitz, M. Golden and H. Georgi Phys.Rev. D36 (1987)1490

[4] E. Farhi and L. Susskind, Phys. Rep. 74 (1981) 277

S. Dimopoulos and L. Susskind, Nucl. Phys. B155 (1979) 237

[5] H. Haber and G. Kane, Phys.Rep.117 (1985) 75

[6] A. Dobado and M.J. Herrero, Phys. Lett. B228 (1989) 495 and B233 (1989) 505

J. Donoghue and C. Ramirez, Phys. Lett. B234 (1990)361

A. Dobado, M.J. Herrero and J. Terrón, Z. Phys. C50 (1991) 205 and Z. Phys. C50 (1991) 465

S. Dawson and G. Valencia, Nucl. Phys. B352 (1991)27

[7] B.Holdom and J. Terning, Phys.Lett. B247 (1990) 88

A. Dobado, D. Espriu and M.J. Herrero Phys.Lett. B255 (1991) 405

M. Golden and L. Randall, Nucl. Phys. B361 (1991) 3

[8] J.M. Cornwall, D.N. Levin and G. Tiktopoulos, Phys. Rev. D10 (1974) 1145

C.E. Vayonakis, Lett. Nuovo Cim.17(1976) 383

[9] B.W. Lee, C. Quigg and H. Thacker, Phys. Rev. D16 (1977) 1519

[10] M.S. Chanowitz and M.K. Gaillard, Nucl. Phys. 261 (1985)379

[11] C. Becchi, A. Rouet and R. Stora, Comm. Math. Phys. 42(1975) 127

[12] G.J. Gounaris, R. Kogerler and H. Neufeld, Phys. Rev. D34 (1986) 3257

[13] Y.P.Yao and C.P. Yuan, Phys. Rev. D38 (1988) 2237 
[14] J. Bagger and C.Schmidt, Phys. Rev. D41 (1990) 264

H. Veltman, Phys. Rev. D41 (1990) 2294

H.J. He, Y.P. Kuang and X. Li, Phys. Rev. Lett. 69 (1992) 2619

W. B. Kilgore, Phys.Lett. B294 (1992) 257

J.F.Donoghue, Phys.Lett. B301 (1993)372

P.B.Pal,Phys. Lett.B321 (1994)229

W.B.Kilgore, Phys. Lett.B323 (1994)161

[15] J. Charap, Phys. Rev. D2 (1970)1115

I.S. Gerstein, R. Jackiw, B. W. Lee and S. Weinberg, Phys. Rev. D3 (2486)1971

J. Honerkamp, Nucl. Phys. B36 (1972)130

Quantum Field Theory and Critical Phenomena, J. Zinn-Justin, Oxford University Press, New York, (1989)

[16] L. Tararu, Phy. Rev. D12 (1975)3351

D. Espriu and J. Matias, Nucl. Phys. B418 (1994)494

[17] Gravitation and Cosmology, S. Weinberg, John Wiley \& Sons (1972)

[18] L. Baulieu and J.Thierry-Mieg Nucl. Phys. B197 (1982) 477

L. Alvarez-Gaumé and L. Baulieu, Nucl. Phys. B212 (1985) 255

L. Baulieu, Phys. Rep. 129 (1985) 1

[19] T. Appelquist and C. Bernard, Phys. Rev. D22 (1980) 200

A. C. Longhitano, Nucl.Phys. B188 (1981) 118

[20] E. Witten,Phys. Lett. B117 (1982)324

[21] L. Alvarez-Gaumé and P. Ginsparg, Nucl.Phys. B262(1985) 439

[22] H.J.He, Y.P.Kuang, and X.Li, Tsinghua preprint TUIMP-TH-94/56, hep$\mathrm{ph} / 9403283$

[23] Tran N. Truong, Phys. Rev. D61 (1988)2526

A. Dobado, M.J. Herrero and J.N. Truong, Phys. Lett. B235 (1990) 134

T.N.Truong, Phys. Rev. Lett. 67 (1991)2260

A. Dobado and J.R. Peláez, Phys. Rev. D47(1992)4883

[24] C.J.C. Im, Phys. Lett. B281 (1992)357

A. Dobado and J.R. Peláez, Phys. Lett. B286 (1992)136

M. J. Dugan and M. Golden, Phys. Rev. D48(1993)4375

[25] M.J. Herrero, E.Ruiz Morales, Nucl. Phys. B418 (1994)431

[26] C.Grosse-Knetter, I.Kuss. Bielefield preprint BI-TP 94/10, hep$\mathrm{ph} / 9403291$ 